mit berufen und befähigt ist, Licht in das hier noch obwaltende Dunkel hineinzubringen, glaube ich mit meinen Ausführungen gezeigt zu haben. Dieses Bemühen gewinnt an Bedeutung, wenn dabei Lichtstrahlen nicht nur auf Probleme der Induskultur, sondern weiterhin auch auf solche der Antike fallen; denn damit rühren wir an die Grundlagen, näherhin an die vorindogermanischen Grundlagen, unserer eigenen europäischen Kultur. $\mathrm{Da}$ vor allem der von Indien ausgehende Völkerforscher zur Aufhellung dieser ebenso interessanten als bedeutungsvollen Fragen sein Scherflein beizutragen in der Lage ist, ist nicht sein eigenes Verdienst. Vielmehr hängt das mit der bekannten Tatsache zusammen, daß dort noch heute vieles von dem lebendige Wirklichkeit ist, was sonstwo seit Jahrtausenden im Schmelztiegel der höheren Kulturentwicklung, sei es weitgehend umgestaltet, sei es völlig, ausgemerzt wurde.

\title{
DIE TÄTIGKEIT DER ARBEITSGEMEINSCHAFT ZUR GEOMOR- PHOLOGISCHEN ERFORSCHUNG DER SCHWEIZER ALPEN 1945
}

Die Arbeit der AGES. ist erfreulicherweise auch im Jahre 1945 gefördert worden. Von ihren bisherigen Resultaten zeugen mehrere im Schweizer Geograph XXII, 1945, publizierte Aufsätze, die das Bestreben erkennen lassen, die Sachforschung mit methodologischer Diskussion zu verbinden, woraus zweifellos die optimalste Klärung der Probleme zu erwarten ist. Die beiden nachfolgenden Berichte über die offiziellen Anlässe der Arbeitsgemeinschaft im vergangenen Jahre erweisen, daß diese auf erfolgverheißendem Wege marschiert.

1. Die Exkursion ins Maderanertal vom 18./19. August 1945.

Ziel der von Dr. E. SCHWABE und PD. Dr. W. STAUB geleiteten Exkursion war die Untersuchung der morphologischen Probleme des Tales und die Diskussion über die Methoden der geomorphologischen Kartierung.

Nach kurzer Orientierung STAUBs über die geologischen Verhältnisse des Gebietes erreichten die Teilnehmer von der Bahnstation Amsteg-Silenen aus, anfangs dem alten Gotthardweg folgend, den Rundhöcker, auf dem die Ruine Zwing-Uri steht (P. 578). Hangwärts, durch eine Erosionsrinne von ihm getrennt (abwechselndes Vorrücken und Abschmelzen des Gletschers), lehnt sich ein wahrscheinlich dem Gschnitzstadium zuzuordnender Moränenwall an, der sich, gekennzeichnet durch Gletscherschliffe und Moränenblöcke, bis nach Frenschenberg (P. 816) verfolgen läßt. Die von hier aus schön überblickbaren Verhältnisse der Konfluenz von Reuß- und Maderanergletscher erweisen sich als recht kompliziert. Der unterste Boden (um Ried, P. 678) oberhalb der Haupttalstufe dürfte dem Talboden von Bristen $(723 \mathrm{~m})$ genetisch entsprechen (Würm?). Unterhalb der Stufe ist die Felssohle unter Alluvium verborgen. Die Bildung des Haupttales der Reuß von Amsteg talabwärts scheint alt zu sein, wie die interglazialen Bergsturzreste von Schattdorf-Haldiberg aus der letzten Zwischeneiszeit beweisen.

Weitere, noch nicht sicher zu klassierende Niveaureste finden sich bei Frenschenberg $(880 \mathrm{~m}$, $960 \mathrm{~m}$ ); Waldiberg (dürfte mit $1360 \mathrm{~m}$ der schönen, wahrscheinlich präglazialen Arnibergterrasse von $1380 \mathrm{~m}$ entsprechen); Rückenegg und Sonnengrat (gehören mit ca. $1900 \mathrm{~m}$ zu deri Niveauresten eines der höchsten Verflachungsgebiete). Nach SCHWABE lassen sich diese Terrassen mit den verschiedenen Leisten im Maderanertal in folgende Niveaus einordnen:

1. Rückenegg . . . . . . . . . Stafelalpen (1900 m) - Alp Gnof (1925 m) - Düssialp $(1959 \mathrm{~m})$.

2. Waldiberg (vermutlich präglazial) . . Geschel $(1520 \mathrm{~m}) \cdot$ - Tritt $(1642 \mathrm{~m})$ - Hüfigletscher (unterhalb P. 1971).

3. Zwischenniveau . . . . . . . Griessernalp $(1305 \mathrm{~m})-$ Balmenegg (1351 m) - Sassegg (ca. $1500 \mathrm{~m}$ ).

4. Frenschenberg . . . . . . Lungenstutz $(1100-1140 \mathrm{~m})$ - Sanderfeld vor Hüfigletscher (ca. $1400 \mathrm{~m})$.

Zur Höhe der aus amphibolitischen Gesteinen bestehenden Terrassenbastion "Auf der Breitlaui» (P. 1133 S. Bristen) zieht von E her eine prächtige, in den weichen Karbonschichten angelegte Verwitterungsterrasse hinauf. Auch in der engen Steilstrecke des Maderanertobels prägt sich der Geschel als Amphibolithärtling aus. Verschiedene wallmoränenartige Bildungen beidseits des Kärstelenbaches im Tobel und oberhalb verlangen genaue Untersuchung (schöner Endmoränenbogen im Balmenwald SW Hotel SAC.).

Während SCHWABE nach dem Nachtessen einen Ueberblick über die Morphologie des Tales gab, wurden während des regnerischen Sonntagvormittags bis $10 \mathrm{Uhr}$ Einzelfragen der morphologischen Kartierung diskutiert.

Bei aufhellendem Wetter zeigte eine Wanderung gegen $\mathrm{Sa}$ (prächtige Stromquelle des auf der Alp Gnof versickernden Wassers; Bergsturzsee von Butzli ohne oberirdischen Abfluß) einen aufschluß- 
reichen Einblick in den Talschluß sowie in den unteren Stufenboden des Brunnitales (ca.1500 m), der dem eiszeitlich vertieften, präglazialen Niveau zu entsprechen scheint.

Auf dem Rückweg über Golzern gab die den Golzersee (P. 1410) stauende Endmoräne Anlaß zur Erörterung der eiszeitlichen Verhältnisse. Da sich in der Moräne nach STAUB typische Windgällenporphyre finden, kann sie nur die Ablagerung eines aus dem großen Windgällenkessel stammenden Hängegletschers sein, dessen Zunge durch den Hauptgletscher in die Ausräumung des Golzersees hinter dem Amphibolithärtling von Geschel verschleppt wurde. Nachdem die neue Golzern-Schwebebahn die Teilnehmer rasch zur Talstraße gebracht hatte, fand die aufschlußreiche Exkursion im Eilmarsch zur Bahnstation ihren Abschluß.

JULES DE ROCHE

\section{Die vierte Tagung der Arbeitsgemeinschaft vom 26. Oktober 1945 in Bern.}

Da der Wert geomorphologischer Forschung, insbesondere der Feldarbeit, weitgehend von der zur Verfügung stehenden Kartengrundlage abhängt, drängt sich dem Geomorphologen eine Orientierung über die in der Schweiz heute zur Verfügung stehenden Kartenwerke auf. Deshalb übernahm es die Arbeitsgemeinschaft zur geomorphologischen Erforschung der Schweizer Alpen, an einer Arbeitstagung vom 26. Oktober 1945 in Bern eine Uebersicht über schweizerische Kartenwerke und die damit im Zusammenhang stehenden Probleme (Photogrammetrie, Kartendarstellung u. a.) zu bieten. Die Schweizerische Landestopographie befaßte sich in zuvorkommender Weise mit der Organisation und Durchführung dieser Tagung, wofür ihr an dieser Stelle bestens gedankt sei.

Nach einer kurzen Begrüßungsansprache des Präsidenten PD. Dr. H. ANNAHEIM, die über Ziel und $Z$ weck der Arbeitsgemeinschaft orientierte, wurde als Verbindungsmann für die Ostschweiz Dr. E. SCHWABE (Zürich) zum vierten Vorstandsmitglied gewählt.

Anschließend referierte Dir. H. SCHNEIDER von der Eidg. Landestopographie über Aufgaben, Organisation und allgemeine Arbeitsprobleme der eidg. Landestopographie. Er gab insbesondere einen Ueberblick über den heutigen Stand der Arbeiten an der Neuen Landeskarte der Schweiz. Durch Uebereinkunft wurde die Grundlagenbeschaffung für dieses Kartenwerk zwischen der Landestopographie und der Vermessungsdirektion aufgeteilt, indem der ersteren die Alpen, der letzteren das Mittelland und der Jura zugewiesen wurde. Die Arbeiten der Eidg. Landestopographie verliefen bisher programmgemäß und werden voraussibhtlich im Jahre 1951 abgeschlossen sein. Die Grundbuchvermessung ist jedoch durch den Aktivdienst in Rückstand geraten, weshalb die bisher veröffentlichtenKarten alpineGegenden wiedergeben.

Daraufhin orientierten Chefing. R. TANK über photogrammetrische und topographische, Chefkartograph BÜHLER über kartographische und reproduktionstechnische Arbeiten an der Neuen Landeskarte. Ihre Ausführungen wurden durch reichhaltige Kartendemonstrationen ergänzt. Die Teilnehmer wurden insbesondere darüber aufgeklärt, welche Kartengrundlagen für die Feldaufnahmen zur Verfügung stehen. Ein Farbenfilm zeigte anschließend die Aufnahmeverfahren unserer neuzeitlichen topographischen Geländevermessung.

Am Nachmittag referierte cand. phil. M. STEIN (Zürich) über geomorphologische Karten und Kartierungsmethoden in den Alpen. Aus seinen reich mit Lichtbildern dokumentierten Ausführungen ging deutlich die Wichtigkeit einheitlicher Kartierung hervor; es zeigte sich, daß jeder Autor eine eigene Darstellungsart verwendete, die auf seine besonderen Bedürfnisse zugeschnitten war.

Abschließend war den Teilnehmern der Tagung Gelegenheit geboten, durch Ingenieure der Eidg. Landestopographie Einblick in die mit Wild-Autographen durchgeführten Auswertungen von erdund luftphotogrammetrischen Kartenaufnahmen zu erhalten.

RUDOLF MERIAN

\section{NEUIGKEITEN}

Au sujet de la géographie du riz. Dans un travail intitulé «Le Riz dans le monde», publié dans la «Revue de Botanique appliquée et d'Agriculture tropicale» en 1912 et 1944 et réimprimé en 1944 (Impr. Ch. Monnoyer, Le Mans) RENE MUSSET nous montre l'importance de cette céréale dans l'économie mondiale. Le Riz est la céréale, non des peuples riches bien nourris, mais des peuples pauvres sousalimentés. Il est moins dispersé, moins universel que le Blé. Hors de l'Extrême-Orient, sa grande zone de culture. L'Afrique soudanienne, autre berceau des espèces spontanées, sa diffusion est liée à des faits de conquête et d'expansion que RENE MUSSET groupe sous un titre évocateur: «Les voyages du Riz». Les Arabes ont porté la précieuses Graminée dans le monde méditerranéen, Syrie exceptée, dans le pourtour africain de l'Océan Indien. Le nom espagnol du Riz (arroz) est d'origine arabe. Les récents progrès du Riz doivent être attribués aux immigrants japonais (Californie, Brésil méridional) et hindous (Est africain anglais et Guyane britannique). Il faut ajouter que le Riz a joué à maintes reprises le rôle de "culture pionnière", préparant la place au Coton (Nord du delta du Nil), à la Luzerne et à la Vigne (Camargue), à l'Hévéa (Sumatra), au Café (arrière pays de Sâo Paulo, du Parana). A l'Asie des moussons reviennent $95,8 \%$ de la production mondiale de $\mathrm{Riz}, 95,4 \%$ de la superficie cultivée (1940: 52000000 ha). L'Inde et la Chine absorbent à elles seules $64,5 \%$ de la production, réunissent $61 \%$ des terres livrées au Riz. La part dans la tiziculture des populations de civilisation européennes se réduit à 2,6\% de la production, $2,3 \%$ de la superficie cultivée (dont $1 \%$ environ pour le Brésil, grâce à des progrès récents). Dans l'hémisphère sud ( $7 \%$ seulement de la superficie réservée au Riz dans le monde) on ne rencontre qu'un seul producteur important, Java. 